\title{
Cooperation scenarios in multi-agent water monitoring platform
}

\author{
Octavian Fratu ${ }^{1}$, Maria Andronache ${ }^{1}$, Ana-Maria Drăgulinescu ${ }^{1}$, Carmen Voicu ${ }^{1}$, Alexandru Vulpe ${ }^{1}$ \\ ${ }^{1}$ Telecommunications Department, University Politehnica of Bucharest
}

\section{Article Info}

Received Nov 12, 2018

\author{
Keyword: \\ surface water vector \\ aerial vector \\ cooperation scenario \\ water monitoring \\ command and control center
}

\begin{abstract}
Monitoring as well as achieving related water quality goals for rivers is a challenge on several scales of both space and time. There are several factors influencing the most effective actions to implement, when it comes to water quality. Collecting water quality data can be challenging and expensive as maintaining specialized equipment can raise costs over time. Within the MultiMonD2 project, a multi-agent platform of micro-laboratories specialized in the water quality monitoring of the Danube river and Delta is being developed. Aerial and surface water vectors are used as carrier systems for sensor-based detection equipment along with a technical solution that allows the cooperation between the robotic vectors. To this end the paper presents architectural solutions for realizing the multi-agent monitoring platform as well as analyzes different possible scenarios for cooperation between aerial and surface water vectors.
\end{abstract}

\section{Corresponding Author:}

First Author,

Telecommunications Department,

University Politehnica of Bucharest,

Splaiul Independenţei no. 313, Sector 6, Bucharest, Romania, Postcode - RO-060042.

Email: octavian.fratu@upb.ro

\section{Introduction}

In the last years, the unmanned aerial vehicles (UAVs) are used in different domains from military applications to personal use. One reason that made this possible is that with the new technologies UAVs with small dimensions can be developed, at a low price and capable of carrying different devices which can be used in applications like agriculture inspections, air surveillance, mapping etc.

One of the challenges of the UAVs is the battery life. In [1] is presented a scenario in which the UAVs are used to improve the wireless service in an urban area. When a specific area needs an improvement of the wireless service the UAV, which has docking stations on the rooftops, receives the command and takes off. The UAV will fly above the served area until enough power is left in order to return at the docking station and recharge. The needed power depends on different factors like the distance between the docking station and served area, the speed, altitude, or weight. There are different solutions which can improve the flight time. One is to use a UAV with a higher battery capacity, but these types of battery are heavier which inevitably leads to an increased weight [2]. A second alternative is to create a docking station where the UAVs can be recharged. This approach was also presented in [1], at the docking station to have another UAVs ready to take the place of the UAV unloaded. But this solution is expensive [3]. Another method is wireless transfer power (WTP). In this way the UAV doesn't need to be recharged. This solution can be split into two main categories: electromagnetic field (EMF) charging and non-EMF charging. EMF charging is based on using magnetic induction or similar to transfer energy. The disadvantages of this solution are that the distance between the UAV and the energy source must be very small, in the order of centimeters, and the received energy is not enough to compensate the energy consumption of the UAV. In the case of the Non-EMF the photo-voltaic (PV) cells are used to charge the UAVs $[4,5]$. 
Another challenge of the UAVs is the autonomous landing. For landing is important to know the locations of the UAV, the docking station and some ideas about the kinematic models [6]. Other essential estimate is the trajectory, for which it must take in consideration the disturbances and uncertainties. As an example, the authors in [7] give a complete system architecture allowing a UAV to land autonomously on a high-speed mobile landing platform under various wind disturbances. For indoor environment, a linear Model Predictive Control (MPC), divided into three main phases can be used, for high-accuracy tracking of a mobile landing platform: target detection, target tracking and autonomous landing [8].

The main contribution of this paper is in formulating cooperation scenarios starting from the aforementioned existing solutions that also fall within the MultiMonD2 project scope, namely to enable a multi-agent platform for the monitoring of the water quality of the Danube river and Danube Delta. Three main scenarios are exposed, related to the interaction between the aerial and surface water vector and what is likely to occur in a real operating environment.

The paper is organized as follows. Section 2 presents a Drone Control Centre as an enabler of the cooperation scenarios and as an architectural solution for realizing the multi-agent monitoring platform for water quality. Section 3 introduces and discusses the three cooperation scenarios, while, finally, Section 4 concludes the paper.

\section{Drone control center}

An architectural solution for realizing the multi-agent water monitoring platform relies on what we call the Drone Control Centre (DCC). DCC has several functional components and is represented in Fig. 1.

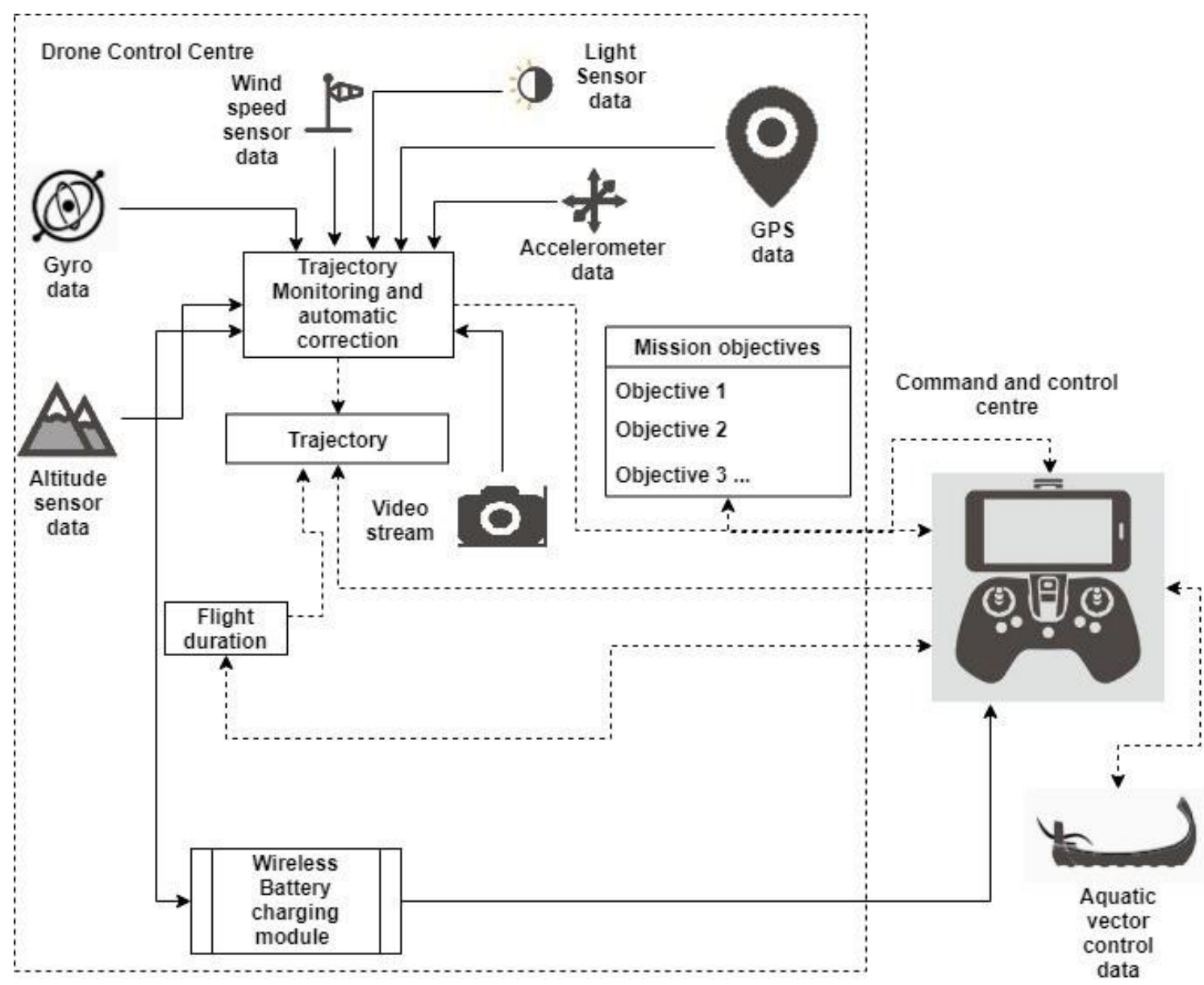

Figure 1. Conceptual scheme of Drone Control Center

The envisioned functioning of the drone control center is described briefly below.

The Trajectory Monitoring and automatic correction block has as inputs the parameters given by the mission objectives established at the Command and Control Centre (CCC). It receives also the data acquired from altitude, gyroscope, light and wind speed sensors, GPS, accelerometer and video monitoring. If altitude sensors, gyroscope, accelerometer and video stream are used to monitor and correct the trajectory of the 
drone, light sensor and wind speed sensor data are collected to add an extra correction and control. For example, light sensor data may be very useful when processing video stream to optimize the path recognition. Another parameter, flight duration, along with the Wireless Battery Charging Module (which both monitors the battery charge and is used for wireless charging) output, adjusts the trajectory of the drone.

The Flight Duration Measuring Block transmits the flight duration to the Command and control Centre (CCC), especially for future optimizations of the wireless charging module and mission objectives. If the battery is under a critical level previously set by the CCC, then the drone automatically changes its trajectory in order to land on the aquatic vector as soon as possible to charge the battery, without human intervention.

\section{Cooperation scenarios}

Within this chapter, a set of scenarios of cooperation between air and surface agents will be defined to achieve specific desiderata. Depending on these scenarios, technical requirements for system communications will also be planned. Both the theoretical development of concepts and proposals for practical schemes that will be further developed will be pursued.

\subsection{Power Supply System for Drone}

Small and medium-sized Unmanned Aerial Vehicle Systems (UAVs) are currently used for autonomous data acquisition tasks, and some examples of their applicability could be: monitoring, surveillance, precision farming, etc. Batteries are the major problem for UAVs to travel long distances because at least half of the battery power should be saved for it to travel back to the recharge point. To create a fully autonomous system, the battery charging procedure must be automated because the drone suddenly lands too low due to a low battery level and requires human intervention to replace the battery or recharge it.

To increase flight time, there are two different possibilities: equipping the drones with a bigger battery or create a base station (where batteries can be automatically changed after landing or where drones will move to automatically recharge the battery).

In this scenario, the appropriate solution is to create a wireless charging panel ground, where the drone can be reached when the battery has a low level. Instead, we need to keep in mind the safety considerations that do not allow this to happen in the event of rain or snow.

The optimal solution is based on the inductive coupling system that consists of two parts: the transmitter side and the receiver side. The primary coil is configured with a plan that contains a series of independent elements, and the secondary coil is placed on the drones. Because drones are usually equipped with intelligent systems such as high definition cameras, proximity or infrared sensors, etc., their viewing area should not be obstructed. The receiving coil is located on the landing platform, thus reducing the distance between the transmission and reception coils. This solution allows an increase of the coupling factor between the coils and thus it is possible to maintain a small and lightweight secondary coil which achieves a high efficiency of the power transmission.

When the drone is trying to land, an automatic procedure begins to determine which of the coils in the landing area is closer to being able to have a maximum coupling factor with the secondary coil. Then, this coil is the only one selected to charge the battery.

Alternative technologies that could be used are based on resonant inductive coupling which, as soon as placement changes more than a few centimeters, offers a more efficient energy transfer.

\subsection{Drone landing on the water surface vector}

The drone and the ground station are equipped with a GPS sensor that has a horizontal position accuracy with an error rate of less than 50\%. So even if the drone approaches the ground station using their own GPS data and ground station GPS data, it is not capable of landing accurately on the ground due to inherent errors in GPS sensors. Therefore, a method should be implemented to detect the exact location of the ground station and to land on it without any collision. A solution to this problem is the use of visually detectable signs. Landing at a short distance from the transmitter creates a sudden drop in charging efficiency, so the solution is to install more transmitters to compensate for inaccurate landing.

A possible algorithm [9] that could be applied is based on minimizing errors and centering the detected image with a video camera located under the drones (or on the adaptive thresholds algorithm). When the drone arrives at the destination, it flies around the area and scans it. The scan area of the image will be restricted to a square when the drone is above the landing site. It will be possible to advance this method by choosing not only a square scan but scanning a square inside which there is a circle. Thus, we will have practically two 
detectable targets that will make it easier for the drones to recognize the landing site. When the landing edges are detected, the drone stops scanning and glides over the target. Additionally, if there is a single circle in the search area, it is assumed to be the target circle and, implicitly, it will be detected that it is the landing position. To filter any image processing error that should distinguish the edges of the square, a sufficient and extra number of iterations will be stored. If we do not have enough iterations, the detected tag is declared as an error and the process is restarted.

Detection should also take into account the different weather conditions, the changes in light intensity and direct sunlight with the shadows caused by the drone.

It should be noted that the velocity profile of the aquatic vector must also be known as well as its trajectory, except for the initial conditions (position and direction). Mathematical operations that underlie this type of algorithm have as main reference [10]. To improve search or tracking, initial experiments should be performed on known parts of the water surface. Successful landing depends on the precise location of the aquatic vector with its coordinates, and essential in this algorithm are the synchronization of the aquatic vector position at the time of acquisition of the image data and calibrating the camera coordinate system to the platform.

\subsection{Directing the water surface vector through the drone}

For this type of scenario, wireless communication will be achieved between the aquatic vector and the drone to avoid frequent obstacles encountered by the aquatic vector.

Ensuring fast communication between mobile agents is a rather difficult issue, due to limited bandwidth and visual obstruction. In particular, the direct line of sight (LOS) is often obstructed by vegetation and other characteristics of the land, and that makes radio communication unreliable and inefficient [11].

It is assumed that the two systems cooperate with each other to transfer data from one location to another, but, if the distance between them gets greater, the wireless connection will become unstable. The first essential steps in establishing communication between the two systems are to identify the optimal distance and the stability of the radio link. Each drone can identify its geographic location using the GPS sensor, therefore, the two systems can measure the physical distance between them.

As a further step, if we want to track stability of wireless communication between them, after determining the optimum distance, the drone should continuously transmit packets and the aquatic vector should calculate their success ratio. If the packet success rate is very low, a solution could be the gradual decrease of the distance between the two vectors until the wireless communication becomes stable. Another idea would be to reduce the packet transmission rate.

There will be four important functions for effective communication between the two systems:

- $\quad$ Controlling the hovering of the drone over the aquatic vector - in the three-dimensional space and for its vertical and horizontal movement

- $\quad$ Controlling the movement of both systems - in the three-dimensional space

- $\quad$ Packet test between the two systems - exchange information on wireless connection stability

- User interaction for both systems - communication with the external controller and real-time transmission of information.

\subsection{Conclusions}

The paper presented scenarios for cooperation in a multi-agent water monitoring platform suitable for the Danube river and Danube Delta. The scenarios stemmed from a devised architectural solution for multi-agent water monitoring, called the Drone Control Center which is used for cooperation between aerial and surface water vectors together with a command and control center. The scenarios target one of the major problems of aerial vectors, namely the battery charge, but also the control of the water surface vector using the aerial one. Therefore, the described scenarios involve cooperation between the two vectors and explore possible ways of realizing it.

The targeted cooperation procedures between vectors opens up a plethora of research challenges, especially in the mathematical geometrical theory of such multi-agent systems which is far from being satisfactory. This can lead to advances with potential applications such as synergetic mission planning, fault tolerance, swarm control, multi-robot path planning, formation generation, formation keeping, exploration and mapping and target tracking, and many others.

Future work will focus on the implementation of the described scenarios, especially on building the power supply system for the aerial vector. 


\section{Acknowledgement}

This work was supported by a grant of the Ministry of Innovation and Research, UEFISCDI, project number 33PCCDI/2018 within PNCDI III.

\section{References}

[1] Galkin, B.; DaSilva, L., "UAVs as Mobile Infrastructure: Addressing Battery Lifetime", arXiv:1807.00996v1, 2018

[2] Campi, T.; Cruciani, S.; Feliziani, M., "Wireless Power Transfer Technology Applied to an Autonomous Electric UAV with a Small Secondary Coil", Energies, 11(2): 352, 2018

[3] Lee, D.; Zhou, J.; Lin, W.T., “Autonomous battery swapping system for quadcopter”, In Proceedings of the 2015 International Conference on Unmanned Aircraft Systems (ICUAS), Denver, CO, USA: 118124,2015

[4] Simic, M.; Bil, C.; Vojisavljevic, V., "Investigation in Wireless Power Transmission for UAV Charging", Procedia Computer Science, 60: 1846-1855, 2015

[5] Kumar, S.; Jayprakash; Mandavi, G.K., "Wireless Power Transfer for Unmanned Aerial Vehicle (UAV) Charging", International Research Journal of Engineering and Technology (IRJET), 04(08): 1939-1942, 2017

[6] Vladareanu, L.; Curaj, A.; Munteanu, R.I., "Complex Walking Robot Kinematics Analysis And PLC Multi-Tasking Control”, Rev. Roum. Sci. Techn. - Électrotechn. et Énerg., 57(1): 90-99, 2012

[7] Feng, Y.; Zhang, C.; Baek, S.; Rawashdeh, S.; Mohammadi, A., "Autonomous Landing of a UAV on a Moving Platform Using Model Predictive Control”, Drones, 2(4): 34, 2018

[8] Macés-Hernández, J. A.; Defaÿ, F.; Chauffaut, C., "Autonomous landing of an UAV on a moving platform using model predictive control", 2017 11th Asian Control Conference (ASCC), Gold Coast, QLD: 2298-2303, 2017

[9] Vladareanu, V.; Munteanu, R.I.; Mumtaz, A.; Smarandache, F.; Vladareanu, L., "The Optimization of Intelligent Control Interfaces Using Versatile Intelligent Portable Robot Platform", Procedia Computer Science, 65: 225-232, 2015

[10] Şandru, O., Vlădareanu, L., Şchiopu, P., Vlădareanu, V., Şandru, A., "Multidimensional Extenics Theory”, U.P.B. Sci. Bull., Series A, 75 (1): 3-12. ISSN 1223-7027, 2013

[11] Wang, H. B.; Zhang, L. L., (2008). Path Planning Based on Ceiling Light Landmarks for a Mobile Robot. 2008 IEEE International Conference on Networking, Sensing and Control, Sanya:1593-1598.

\section{BIBLIOGRAPHY OF AUTHORS}

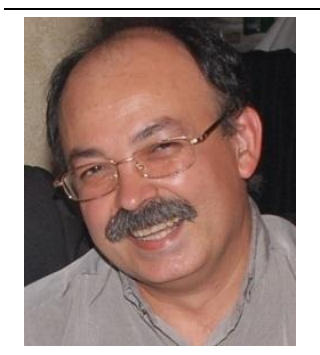

\footnotetext{
Octavian Fratu received the $\mathrm{PhD}$ degree in Electronics and Telecommunications from the University "Politehnica" of Bucharest, Romania in 1997. He achieved postdoctoral stage as senior researcher in 3rd generation mobile communication systems, based on a research contract between CNET-France, ENS de Cachan - France and Universite Marne la Vallee - France. He is currently professor in Electronics, Telecommunications and Information Theory Faculty. His research interests include Digital Mobile Communications, Radio Data Transmissions, Mobile Communications and Wireless Sensor Networks. His publications include more than 200 papers published in national or international scientific journals or presented at international conferences. He participated as director or collaborator in many international research projects such as "Optimization and rational use of Wireless Communication Bands (ORCA)" (NATO Science for Peace research project, 2013-2016), „Reconfigurable Interoperability of Wireless Communications Systems (RIWCoS)" (NATO Science for Peace research project, 2007-2010), „REDICT-Regional Economic Development by ICT/New media clusters” (FP7 CSA project, 20082009), "eWALL for Active Long Living - eWALL" (FP7 IP project, 2013-2016) and other. Currently he is leading the national funded "Integrated Software Platform for Mobile Malware Analysis (ToR-SIM)" no. 5Sol/so17 project and the communication subproject of MultiMonD2 complex project no. 33PCCDI/2018.
} 


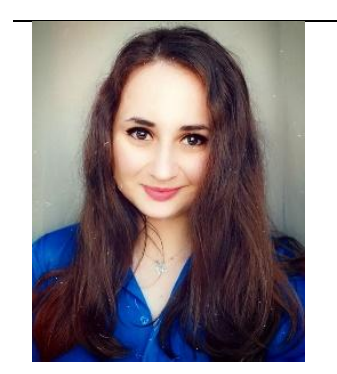

Maria Andronache received her B.Sc in Telecommunication Technologies and Systems at University POLITEHNICA of Bucharest, Electronics, Telecommunications and Information Theory Faculty, in 2018. Since then, she is a student in master's degree Programme in Mobile Communications. She is a participant in communication subproject of MultiMonD2 complex project no. 33PCCDI/2018. Her research interests include the next generation wireless communication, IoT, Wireless Sensor Networks, Network and Services Security, Machine Learning.

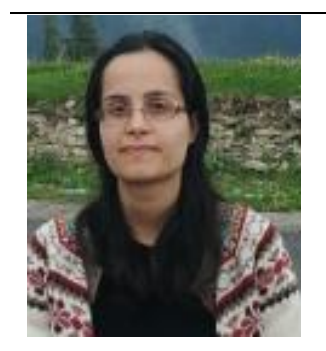

Ana Maria Drăgulinescu received her B. Eng. in Telecommunication Systems and Technologies (2014) and her M. Eng in Multimedia Technologies in Content Production for Audiovisual and Communication (2016) from Faculty of Electronics, Telecommunication and Information Technology. Since 2016, she is PhD student with a thesis focused on developing and enhancing sensors and IoT systems based on LPWAN technologies. Since 2017, she is Research and Teaching Assistant in Telecommunication Department of University Politehnica of Bucharest. Her main interests are automation, sensor systems and networks, Internet of Things and Internet of Medical Things, LPWAN communication technologies.

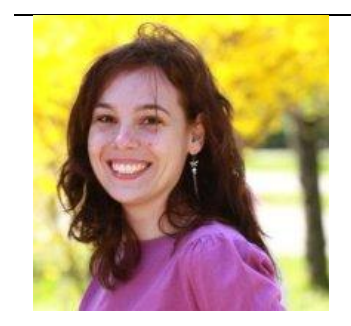

Carmen Voicu received her M.Sc in Mobile Communications in 2011 and the $\mathrm{PhD}$ degree, in the area of multiuser MIMO and its applications in wireless communications, in 2014. She has been Assistant Professor (2011-2014) and from 2014 she is Lecturer at University POLITEHNICA of Bucharest, Electronics, Telecommunications and Information Theory Faculty, Telecommunications Department. She is a participant in several research projects focused on wireless networks with an activity related to signal processing. Her domains of interest are Multiple Access Systems \& Techniques, Analog and Digital Transmission Systems, Channel Coding for Wireless Systems.

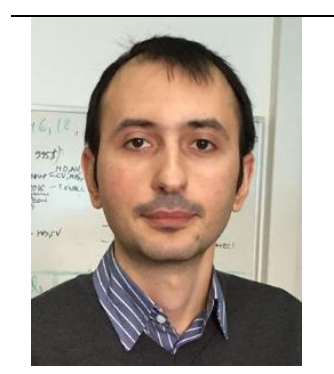

Alexandru Vulpe received the $\mathrm{PhD}$ degree in Electronics, Telecommunications and Information Technology from the University POLITEHNICA of Bucharest, Romania in 2014, and is currently a lecturer with the same institution. His research interests include, among others, Wireless Sensor Networks, eHealth, Mobile Communications, Security, Quality-of-Service, Radio Resource Management, Mobile Applications. His publications include more than 50 papers published in journals or presented at international conferences. He participated as a manager or researcher in a number of national or international projects in the area of eHealth, Security and Internet of Things such as „Platform for Studying Security in Internet-of-Things (PaSS-IoT)”, UPB Excellence Grants project (2016-2017), „eWALL - eWall for Active Long Living” (FP7 project, 2013-2016), “Optimization and Rational Use of Wireless Communication Bands (ORCA)", NATO Science for Peace project (20132015), "Terms of Reference - Integrated Software Platform for Mobile Malware Analysis (ToR-SIM)", UEFISCDI Solutions project (2017-2020). 